\title{
Electronically driven phase transitions in a quasi-one-dimensional adsorbate system
}

\author{
P. Amann ${ }^{1}$, M. Cordin ${ }^{1}$, Ch. Braun ${ }^{1}$, B.A.J. Lechner ${ }^{1}$, A. Menzel $^{1}$, E. Bertel ${ }^{1, a}$, C. Franchini ${ }^{2}$, R. Zucca ${ }^{3}$, \\ J. Redinger ${ }^{3}$, M. Baranov ${ }^{4}$, and S. Diehl ${ }^{4}$ \\ 1 Institute of Physical Chemistry, University of Innsbruck, Innrain 52a, 6020 Innsbruck, Austria \\ 2 Faculty of Physics, University of Vienna, Sensengasse 8, 1090 Vienna, Austria \\ 3 Institute of Applied Physics and Center for Computational Materials Science, Vienna University of Technology, Gußhausstr. \\ 25-25a, 1040 Vienna, Austria \\ 4 Institute of Quantum Optics and Quantum Information - Austrian Academy of Sciences, Technikerstr. 21a, \\ 6020 Innsbruck, Austria
}

Received 30 September 2009 / Received in final form 11 December 2009

Published online 2 February 2010 - (c) EDP Sciences, Società Italiana di Fisica, Springer-Verlag 2010

\begin{abstract}
A quasi-1D system is prepared using the Pt(110) surface as a template. The electronic surface resonance structure is studied by angle-resolved photoemission spectroscopy for the clean surface as well as for different Bromine coverages. A Fermi surface mapping reveals saddle points at the Fermi level in the interior of the surface Brillouin zone. Correspondingly, a maximum in the static response function $\chi(q, 0)$ at the connecting vector $q$ is expected. With $1 / 2 G_{x}<q<2 / 3 G_{x}$ one observes indeed a 3-fold periodicity around defects and a 2-fold periodicity at low temperature for $\Theta_{\mathrm{Br}}=0.5 \mathrm{ML}$. Cooling of a defect-free $c(2 \times 2)-\mathrm{Br} / \mathrm{Pt}(110)$ preparation counter-intuitively results in a loss of long-range order. Motivated by DFT calculations this is attributed to an anomalous order-order phase transition into the $(2 \times 1)$ phase accompanied by intense, strongly anisotropic fluctuations within a temperature range of $\sim 200 \mathrm{~K}$. The peculiar behaviour is rationalised in terms of a competition between inter-adsorbate repulsion and an adsorbate triggered $2 k_{F}$ interaction in the substrate.
\end{abstract}

\section{Motivation}

In quasi-one-dimensional (quasi-1D) materials the reduced screening leads to enhanced electronic correlation. As a consequence, a variety of electron-electron and electron-phonon interactions give rise to ordering phenomena, such as charge, spin, and orbital ordering. In addition, there is a strong tendency for electron localization. This arises on the one hand from the electronic Coulomb interaction, which can give rise to a Mott-Hubbard insulator transition, on the other hand from defect-induced Anderson localization [1]. The latter is promoted in quasi1D systems for two reasons: first, in the entropy-energy balance of defect formation, entropy takes the lead as the dimensionality is lowered, favouring the proliferation of defects; second, the lower the connectivity of a system, the easier is the disruption of well-defined phase relations. Delocalised-localised phase transitions in addition to the various ordering phase transitions are therefore contributing to the exceptionally rich phase diagram of quasi-1D systems. But this is not yet the complete story. Due to the variety of phases and of electronic interactions the Gibbs Free-Energy surface develops several local minima in phase space, which often are separated only by small

\footnotetext{
${ }^{a}$ e-mail: Erminald.bertel@uibk.ac.at
}

barriers. If the thermal energy is comparable to these barriers, the systems may fluctuate both in space and time. In some cases the barrier is even lower than the zeropoint energy, in which cases quantum fluctuations may occur. Since in low-dimensional systems such fluctuations are not confined to extremely narrow parameter ranges, as is the case in higher dimensions (compare for instance the well-defined critical point for the liquid-vapour transition of water), one might consider fluctuating low-D systems as a peculiar state of matter. As fluctuations give rise to novel interactions, the corresponding materials properties can be significantly different from those appearing in the static case. For example, anti-ferromagnetic fluctuations provide an effective singlet pairing potential with d wave symmetry [2] and may be at the origin of high-temperature superconductivity. Critical Casimir forces [3] are another example illustrating this point. Finally, at least as far as quantum critical fluctuations are concerned, it appears that systems frequently avoid this exotic state by escaping into still another stable phase. Unconventional superconductivity [4] might be one of these cases. Given the fact that electronic correlation in low-D systems is not very well understood, this provides ample motivation to investigate a variety of model systems, where one can hope to disentangle some of the mechanisms. In the present study 
we introduce a quasi-1D surface system on a metallic substrate. We report a highly anomalous phase transition, which is entirely classical and weakly first-order, but nevertheless exhibits quasi-critical fluctuations over an extremely large temperature range. This behaviour stems from a competition between indirect adsorbate-adsorbate interactions and an electronic $2 k_{F}$ interaction driving the system into a charge density wave phase.

\section{Experimental approach and computational methods}

Apart form a host of materials, in which 2D or 1D subunits are coupled into a 3D material, there is increasing activity of fabricating quasi-1D materials on surfaces. This renders such systems accessible for the whole toolbox of surface science and allows in particular direct space imaging of phase transitions and their microscopic dynamics by scanning probe microscopy. The majority of approaches are focused on semiconductor surfaces with embedded metal chains. A severe problem here arises from the control of defects and from the rather intricate electronic structure. It is hard to keep the defect concentration on semiconductor surfaces low enough to avoid the behaviour of quasi-1D systems to be totally controlled by the presence of defects.

Preparation techniques are improving though, and it might be possible to obtain reasonably perfect surfaces in the near future [5]. A second difficulty is associated with the interpretation of the band structure as obtained for instance by angle-resolved photoemission spectroscopy (ARPES), since there is some controversy about the localization of the quasi-1D electronic states, which constitute the relevant parts of the Fermi surface [6]. While it is tempting to identify them as metal chain states, DFT calculations suggest an alternative assignment as step-edge states. Furthermore, the nature of the phase transitions observed is highly controversial $[7,8]$. Setting the details aside, however, there exist already excellent studies of structural transitions on such surfaces $[9,10]$.

The present study reports a complementary approach: it starts from electronic surface states on metallic surfaces. Imposing a 1D nanostructure on the surface then renders them quasi-one-dimensional, i.e. provides a sufficiently strong difference between the parallel $\left(t_{\|}\right)$and perpendicular $\left(t_{\perp}\right)$ transport matrix element to obtain quasi-1D behaviour. The advantage in using metallic surfaces is the high perfection with which such surfaces can be prepared. Hence one can study the behaviour as a function of the defect concentration starting from phases with defect concentrations as low as $10^{-5} \AA^{-2}$. The price to be paid is the comparatively strong interaction of metal surface states and in particular surface resonances with the bulk, which causes a considerable broadening of quasi-particle peaks in angle-resolved photoemission spectroscopy (ARPES). It is therefore more difficult to determine the precise band dispersions, Fermi-surface shapes and gap parameters as compared to the semiconductor templates.

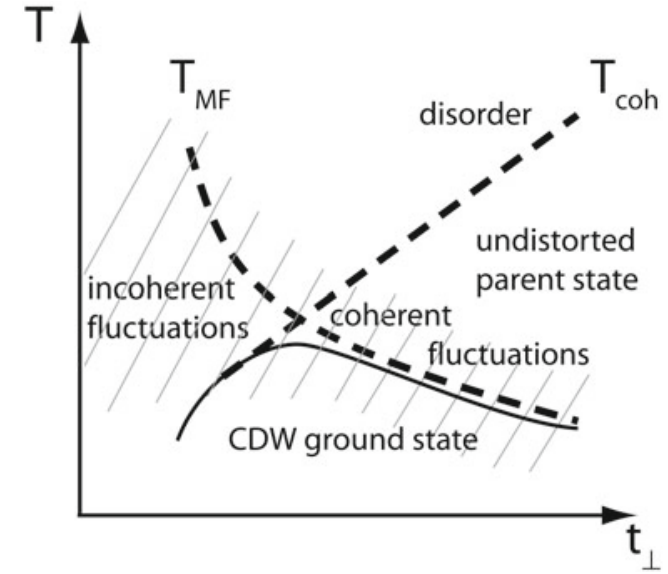

Fig. 1. Generic phase diagram of quasi-1D materials with a CDW groundstate. $\mathbf{t}_{\perp}$ is the coupling between individual $1 \mathrm{D}$ subunits. Large coupling (right side) renders the material 2D, resp. 3D and thus suppresses the mean field temperature $T_{M F}$ for the Peierls transition. Small coupling (left side) reduces the interaction between the subunits and therefore the temperature $T_{c o h}$, at which the coherence breaks down. On the left side, the CDW phase develops without phase correlation between the different subunits. On the right side, a coherent 2D (3D) CDW phase is formed, which melts via coherent fluctuations.

Apart from experimental considerations, the existing data in the literature suggest that the transversal coupling between the 1D moieties is generally weaker on semiconductor substrates, which is of course hardly surprising. Taking a generic phase diagram of quasi-1D systems as a guide line [11], one may therefore expect to explore qualitatively different regions of the phase diagram as illustrated in Figure 1.

Based on these considerations we decided to study fcc (110) surfaces of transition metals, which provide the required anisotropy. Since despite this anisotropy a considerable curvature (warping) of the surface-state Fermi surfaces is likely, given the coupling through the metallic bulk, Fermi surface nesting might not be very pronounced. To nevertheless obtain a high electronic susceptibility, which is the prerequisite for electronically driven phase transitions, a high density of states (DOS) at $E_{F}$ is indispensable. Such a high DOS can be provided by Tamm surface states, which split off the d band edges [12]. We therefore focused our investigation on $\mathrm{Pt}(110)$, where the $\mathrm{d}$ band is almost completely occupied and we expected to find surface resonances derived from the top of the $d$ bands. The $(1 \times 2)$ missing-row reconstruction of the clean surface increases the distance between the close packed atom rows on the surface to two lattice constants, i.e. to $7.82 \AA$, thus enhancing the anisotropy. In order to manipulate the population and hence the Fermi surface of the surface resonances we chose halogen adsorption, assuming that the charge transfer into the halogen p shell would depopulate the surface resonances. While this simple concept did not work out as anticipated, halogen adsorption 

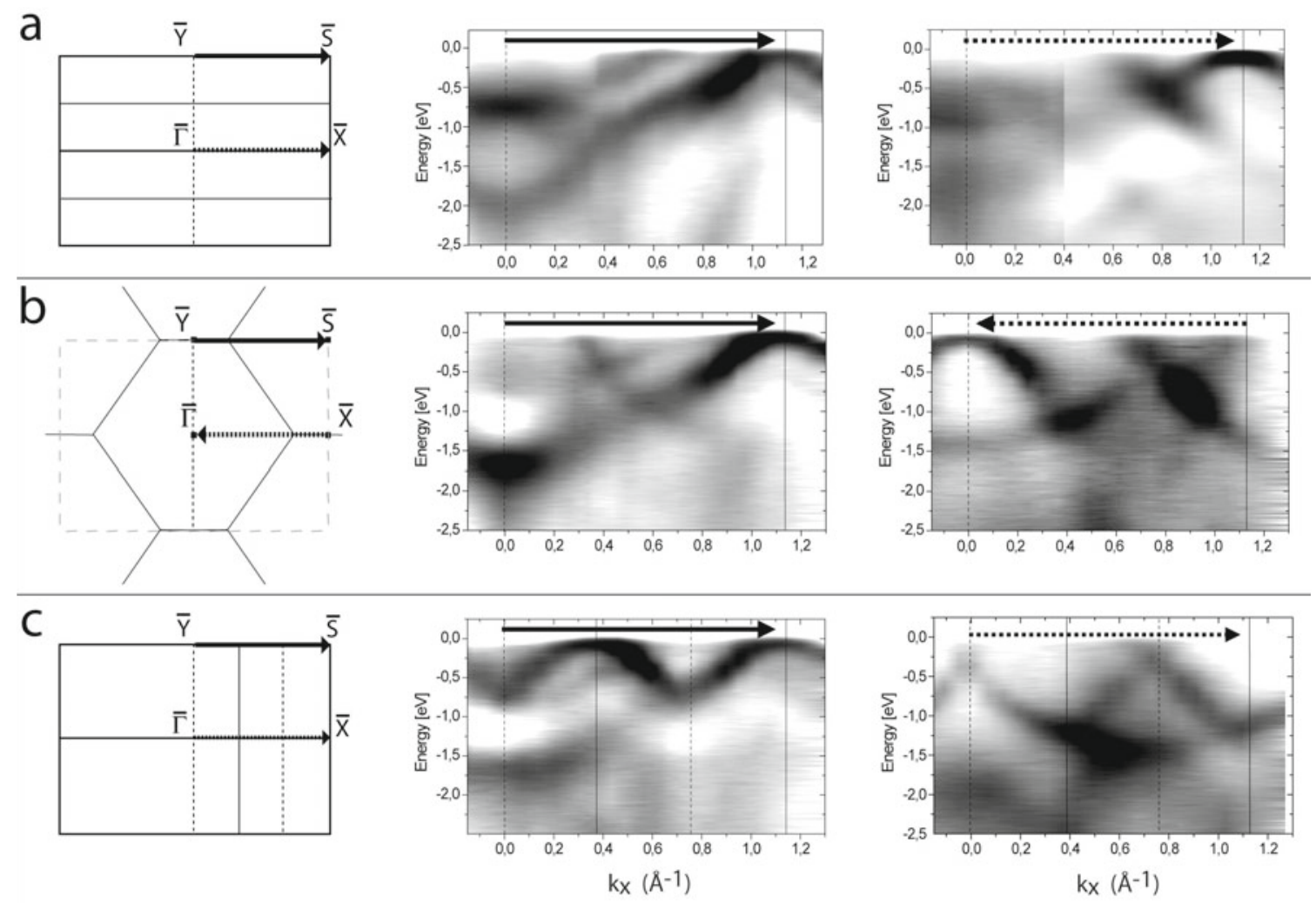

Fig. 2. (a) Surface Brillouin zone (SBZ) of the clean Pt(110)- $(1 \times 2)$ m.r. surface and ARPES intensity plots for the $\bar{Y} \bar{S}$ and the $\bar{\Gamma} \bar{X}$ direction. The $\bar{S}$ feature located at $E_{F}$ appears with similar intensity at the symmetry equivalent $\bar{X}$ point. (b) Surface Brillouin zone (SBZ) of the $c(2 \times 2)-\mathrm{Br} / \mathrm{Pt}(110)$ surface and ARPES intensity plots for the $\bar{Y} \bar{S}$ and the $\bar{\Gamma} \bar{X}$ direction. Here, the $\bar{S}$ feature is mapped onto the $\bar{\Gamma}$ point. (c) Surface Brillouin zone (SBZ) of the $(3 \times 1)-\mathrm{Br} / \mathrm{Pt}(110)$ surface and ARPES intensity plots for the $\bar{Y} \bar{S}$ and the $\bar{\Gamma} \bar{X}$ direction. In this case the two lines in the SBZ are not related by symmetry, but both reflect the threefold symmetry of the surface. The $\bar{S}$ feature is back-folded to the new $(3 \times 1)$ SBZ boundary.

nevertheless turned out to be a lucky choice, as will become clear below.

First-principles density functional theory (DFT) calculations were carried out by means of the Vienna ab initio Simulation Package (VASP) [13]. Although all results were obtained by using both, the generalised gradient approximation (GGA) according to Perdew and Wang (PW91) [14] and the local density approximation (LDA) as given by Perdew-Zunger (Ceperly-Alder) [15,16], the figures given here refer to the latter, since LDA performs better for the $5 d$ series [17]. In the calculations, repeated slabs with $11 \mathrm{Pt}$ substrate layers separated by $14 \AA$ vacuum have been used. Further computational details are given in reference [18].

\section{ARPES results}

Figure 2 shows a collection of ARPES intensity plots as a function of $k_{x}$, i.e. parallel to the close-packed rows (viz. (11̄0) direction). In Figure $2 \mathrm{a}($ clean $\mathrm{Pt}(110)-(1 \times 2)$ m.r. $)$ a band disperses from about $-2 \mathrm{eV}$ at $\bar{Y}$ to $E_{F}$ at $\bar{S}$. At $\bar{S}$ it becomes very intense and the bandwidth is significantly reduced. This is reminiscent of the $\bar{S}$ surface state on $\mathrm{Ni}(110)$ investigated by Eberhardt et al. [12] long ago. If the band has surface resonance character, it should be back-folded according to the symmetry of the reconstructed surface. The missing-row reconstruction introduces a new surface Brillouin zone (SBZ) boundary in x-direction between $\bar{S}$ and $\bar{X}$, rendering these two points symmetry equivalent. The ARPES intensity plot for the line $\bar{\Gamma}-\bar{X}$ does indeed show an intense replica of the feature at $\bar{S}$, thus confirming its resonance character.

Further tests for the surface localization of the observed ARPES features stem from the adsorption of Bromine. Br adsorption lifts the missing-row reconstruction and depending on coverage, completely different surface symmetries are obtained. Figure $2 b$ shows ARPES plots for the quasi-hexagonal structure obtained at $\Theta_{\mathrm{Br}}=$ $0.5 \mathrm{ML}$ (one monolayer corresponds to the areal density of $\mathrm{Pt}$ atoms on the $\mathrm{Pt}(110)-(1 \times 1)$ surface $)$. Here, the $\bar{S}$ point is equivalent to the $\bar{\Gamma}$ point and accordingly the $\bar{S}$ surface resonance is back-folded to $\bar{\Gamma}$ in the ARPES plot. Increasing the coverage to $\Theta_{\mathrm{Br}}=0.67 \mathrm{ML}$ imposes a threefold symmetry on the surface as illustrated in Figure 2c. Now the $\bar{S}$ feature appears at the equivalent point at $k_{x}=0.35 \AA$ on the $\bar{Y}-\bar{S}$ line. Moreover, the whole band is seen to reflect the new periodicity revealing its sensitivity to the additional surface-potential Fourier component. The $\bar{\Gamma}-\bar{X}$ line is no longer symmetry equivalent, yet the 


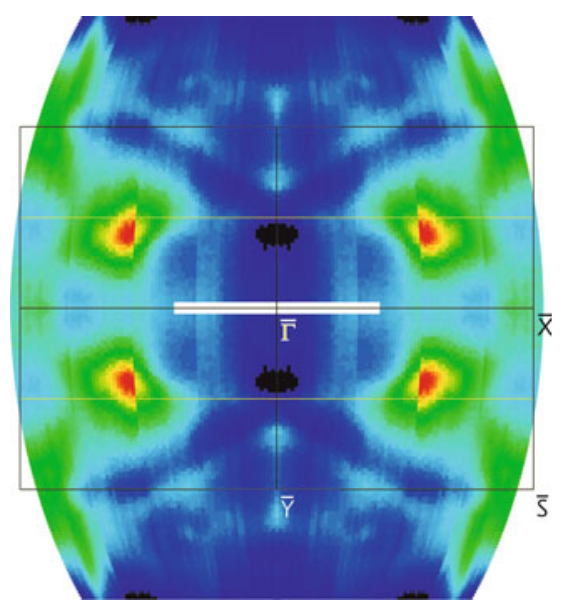

Fig. 3. ARPES intensity map recorded at constant energy within an energy window of $200 \mathrm{meV}$ centred at the Fermi energy. The contours of the clean Pt(110) SBZ are shown as well. Blue corresponds to low, red to high count rates. The blue basins above and below $\bar{X}$ are hole pockets, the blue basin to the left of $\bar{X}$ is an electron pocket.

corresponding ARPES plot, too, shows a band which exhibits the threefold symmetry, indicating that this band has also surface resonance character. Note that it crosses the Fermi level and therefore contributes to the DOS at $E_{F}$ as well. As discussed previously, the $\bar{S}$ surface resonance is associated with a saddle point topology. In an ideal 2D system such a topology would give rise to a divergence in the DOS, in a real system it will at least make a strong contribution to it. Figure 3 shows a map of the ARUPS intensity at $E_{F}$ for the missing-row reconstructed $\mathrm{Pt}(110)-(1 \times 2)$ surface. The most striking feature is a high-intensity spot in the interior of the SBZ around $\left(k_{x}, k_{y}\right)=(0.65,0.33) \AA^{-1}$. From a detailed inspection one obtains also a saddle point topology. Therefore it is fair to say that saddle points with surface resonance character dominate the DOS close to $E_{F}$. An additional observation, however, is important in the present context: The contours seen in Figure 3 have a significant curvature and no pronounced Fermi surface nesting can be derived from this Fermi intensity map. Finally, we remark that the intensity distribution is rather extended in $k$ space and does not reflect clear Fermi surface contours. This is due to the comparatively flat dispersion of the $d$ derived bands - particularly at the upper edge of the $d$ band manifold - as compared to the strongly dispersive $s, p$ bands in other systems, e.g. noble metals. In addition, correlation effects lead to a mass renormalization of the bands close to $E_{F}[19]$, reducing their slope even more. However, a detailed study of the band topology throughout the SBZ revealing position and shape of electron and hole pockets allows a reasonably good interpolation of at least the most relevant parts of the Fermi surface.

In Figure 4 the relation of the ARUPS features to the bulk band structure is analysed for the case of the $\bar{S}$ derived feature. Figure 4 a shows again the ARPES intensity plot, while in Figure $4 \mathrm{~b}$ the $k_{z}$ integrated (linear) bulk DOS is shown. It is obtained as $\int E\left(k_{x}, k_{y}, k_{z}\right) d k_{z} d E$ $k_{z}$

where $E\left(k_{x}, k_{y}, k_{z}\right)$ is the $3 \mathrm{D}$ bulk band structure, $d E$ is a predefined energy interval $(d E=100 \mathrm{meV})$ and the integration is from the central plane of the bulk BZ $\left(k_{z}=0\right)$ to the edge of the BZ. Obviously, the linear DOS will be high, wherever $\nabla_{k_{z}} E(\vec{k}) \rightarrow 0$. These are precisely the points, where one can expect Tamm states to be formed, since at the surface the corresponding degenerate wave functions $\Psi\left(k_{\|}, k_{z}\right)$ can be combined to form localized wave packages. Figure 4 shows clearly that the $\bar{S}$ feature does indeed derive in this sense from a bulk band edge. Due to the $(1 \times 2)$ reconstruction it also appears at $\bar{X}$. Using a similar analysis for other bands which are back-folded according to the surface symmetry one can clearly discriminate the back-folded from the original surface resonance, which is not always possible, if only the intensity is considered.

Similar observations apply for the ARPES results of the $c(2 \times 2)$ phase at $\Theta_{\mathrm{Br}}=0.5 \mathrm{ML}$. Here, too, the Fermi surfaces display an appreciable curvature, making nesting comparatively inefficient. Interestingly, both, the saddle point at $\bar{S}$ and the one in the interior of the SBZ remain right at $E_{F}$, despite the considerably different surface geometry and composition. Apparently, the saddle points pin the surface resonance relative to $E_{F}$.

In summary, the Fermi surfaces provide no unique evidence for the quasi-1D character of the system. However, the saddle points found in the interior of the SBZ provide a very high DOS at $E_{F}$. Accordingly one can expect a maximum in the static electronic response function $\chi(q)$ for the connecting vector $q=2 k_{F}$ rendering the system unstable towards perturbations with a similar periodicity [20]. In the following we focus on the $\mathrm{c}(2 \times 2)-\mathrm{Br} / \mathrm{Pt}(110)$ structure, since it is the phase diagram of the latter, which will be discussed further below.

\section{Quasi-1D behaviour of the $\mathrm{c}(2 \times 2)-\mathrm{Br} / \mathrm{Pt}(110)$}

First indications of quasi-1D behaviour are provided by defective preparations of the $c(2 \times 2)$ structure as shown in Figure 5. Around defects, the $c(2 \times 2)$ order is destroyed and an apparent $(1 \times 1)$ structure is visible, albeit with a clear intensity modulation. The intensity modulation is obviously associated with the defects and signals a perturbation, which propagates as a plane wave, as opposed to circular wave propagation in an isotropic medium. The precise interpretation of the STM image, however, is not straightforward, since a $\mathrm{Br}$ coverage of $0.5 \mathrm{ML}$ cannot give rise to a $(1 \times 1)$ structure. Furthermore, the contrast in the STM image is entirely due to $\mathrm{Br}$ atoms in short-bridge sites $[18,21]$, hence one should expect a uniform contrast. Closer examination reveals that the contrast results from fluctuations of the $\mathrm{Br}$ atoms: on the defective surface the $\mathrm{Br}$ atoms are mobile and visit every possible lattice site. Since the STM sampling time is large as compared to the $\mathrm{Br}$ hopping time scale, a 

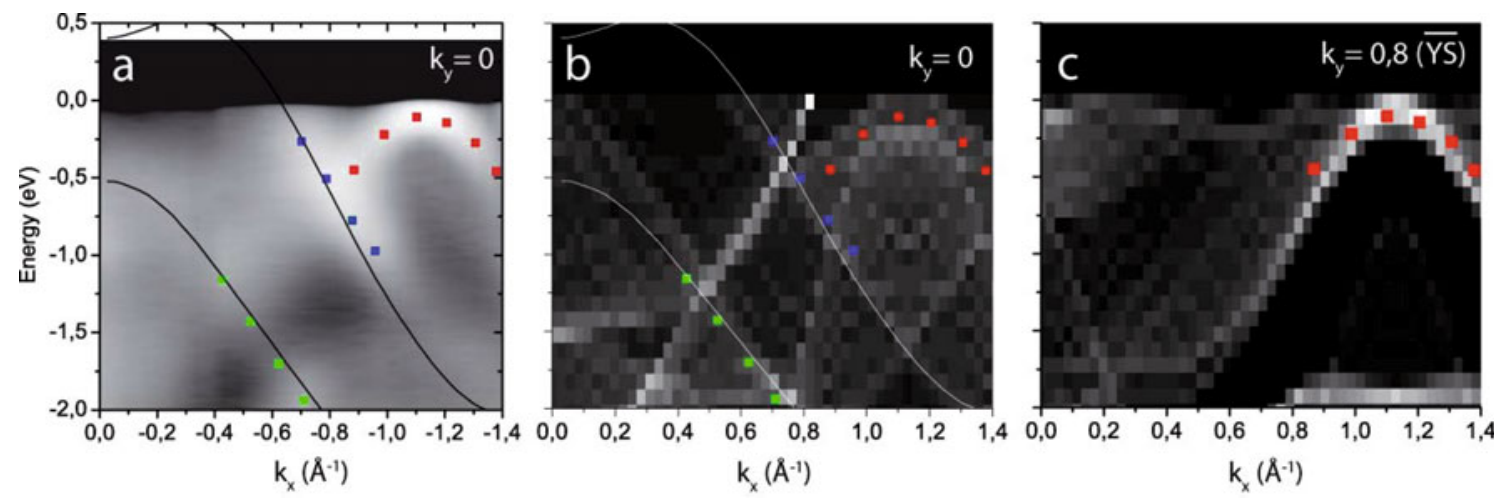

Fig. 4. (a) ARPES intensity recorded on $\operatorname{Pt}(110)-(1 \times 2)$ m.r. along the line $\bar{\Gamma} \bar{X}$. Coloured symbols refer to peak positions determined from the electron distribution curves (EDCs). Thin black lines mark the direct transitions expected from DFT band structure calculations (using $h \nu=21.2 \mathrm{eV}$ ). (b) Linear bulk DOS calculated as described in the text for the line $\bar{\Gamma} \bar{X}$. White corresponds to high, black to low DOS. (c) Linear bulk DOS calculated for the line $\bar{Y} \bar{S}$. The feature seen in the ARUPS data at $\bar{X}$ corresponds to an extremely high linear DOS at $\bar{S}$ deriving from a band, which at $\bar{S}$ exhibits only little $k_{z}$ dispersion. It appears at $\bar{X}$, because the $\bar{X}$ point is equivalent to the $\bar{S}$ point on the reconstructed surface.
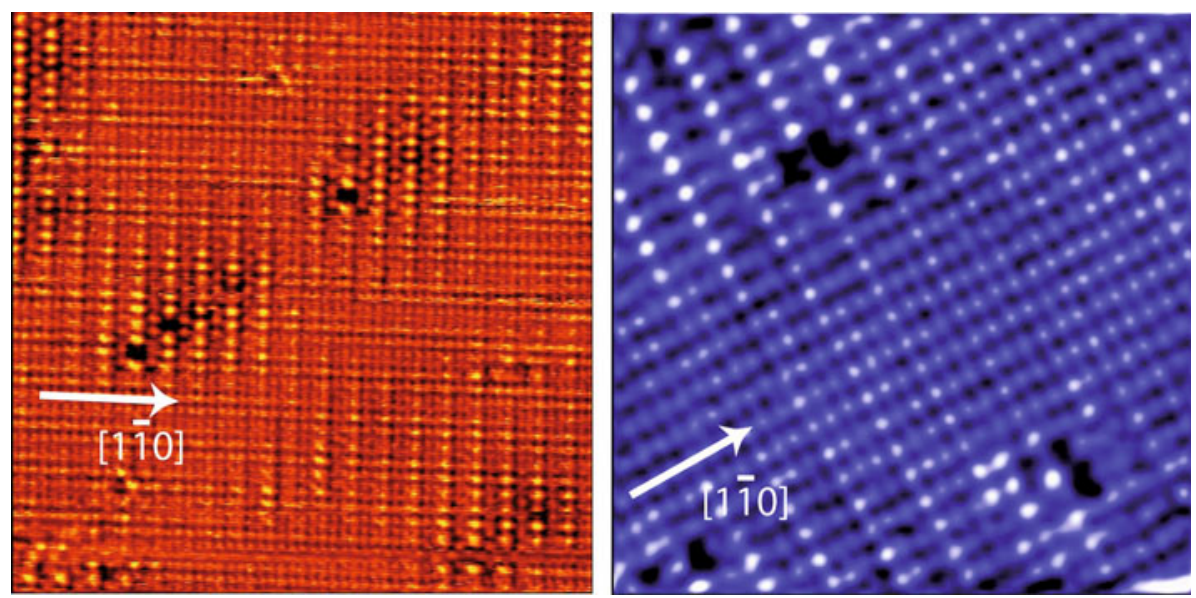

Fig. 5. Scanning tunnelling microscopy images of a defective preparation of $0.5 \mathrm{ML} \mathrm{Br}$ on $\mathrm{Pt}(110)$ recorded at room temperature. Dark spots are defects (presumably Pt atom vacancies). The defects induce a perturbation, which propagates as a plane wave with a periodicity of three nearest-neighbour distances in the [110] direction. For further details see text.

$(1 \times 1)$ structure is obtained as a time average. The intensity modulation arises from different residence times of the $\mathrm{Br}$ atoms in the individual short-bridge sites. Longer residence times correspond to brighter contrast. Interestingly, the intensity is modulated with a period of three nearest-neighbour distances in $[1 \overline{1} 0]$ direction along the close-packed rows, although the $\mathrm{Br}$ coverage is 0.5 ML. The threefold periodicity results apparently from the interaction with the substrate. As previously discussed, we argue that the substrate is close to forming a charge density wave (CDW) [21]. Note that the connecting vector of the saddle points shown in Figure 3 is $q_{x}=1.3 \AA^{-1}$. Hence $1.12 \AA^{-1}=G_{x} / 2<q_{x}<2 G_{x} / 3=1.49 \AA^{-1}$ where $G_{x}$ is a reciprocal surface lattice vector in the close-packed row direction.

Further evidence of 1D behaviour stems from a peak profile analysis of low-energy electron diffraction spots as a function of temperature [22]. Diffraction spot profiles of fluctuating materials are typically composed of a Gaussian contribution, which is proportional to the square of the long-range order parameter, and a Lorentzian contribution, which arises from short-range correlation in the fluctuating system. The intensity of the Lorentzian is proportional to the intensity of the fluctuations and - equivalently - to the generalized susceptibility of the material, while the FWHM scales roughly as the inverse square of the correlation length. Figure 6 shows the intensity of the Lorentzian contribution to the half-order spot profile as a function of temperature measured parallel and perpendicular to the close-packed row direction, respectively.

In the perpendicular measurement the Lorentzian profile appears at around $350 \mathrm{~K}$, reaches a maximum at $370 \mathrm{~K}$ and drops off towards higher temperatures. This is the typical fingerprint of critical scattering at a continuous order-disorder transition, as has been repeatedly observed in adsorbate systems [23]. In contrast, if examined in the direction parallel to the close-packed rows one finds a rather different behaviour: correlated fluctuations set in as the critical temperature is approached from above, just 


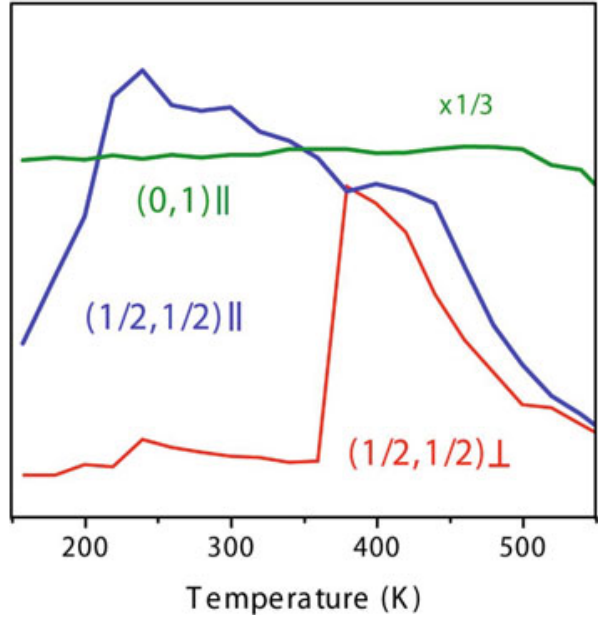

Fig. 6. Lorentzian contribution to the spot profile of the $(1 / 2,1 / 2)$ and the $(0,1)$ spot of the $c(2 \times 2)$ LEED pattern as a function of temperature. The blue (red) line indicates the Lorentzian contribution to the half-order spot as measured parallel (perpendicular) to the close-packed row direction, the green line shows the Lorentzian contribution to the integerorder spot in the parallel profile.

as in the previous case. However, below $T_{c}$ the fluctuation intensity even grows upon cooling. Only for $T<220 \mathrm{~K}$ the Lorentzian intensity decreases, actually together with the long-range order, thus signalling a total loss of order in the system. The striking anisotropy in the fluctuation behaviour as a function of $T$ is a clear indication of quasi-1D behaviour. The $(0,1)$ spot, which stems predominantly from scattering on substrate atoms, exhibits a large Lorentzian contribution throughout the $T$-range examined in the analysis.

Finally, a strong anisotropy is seen in the repulsive interaction of $\mathrm{Br}$ atoms on $\mathrm{Pt}(110)$. DFT calculations show a very weak repulsion of $\mathrm{Br}$ atoms separated by $3.92 \AA$ across the close-packed row direction. Conversely, the repulsion between $\mathrm{Br}$ atoms along the close-packed atom row increases dramatically for separations below $5.54 \AA$. In fact, it is impossible to compress the Br layer on $\mathrm{Pt}(110)$ to below this distance. Rather, the otherwise energetically less favourable long-bridge sites are occupied in the $(3 \times 1)$ $\left(\Theta_{\mathrm{Br}}=0.67 \mathrm{ML}\right)$ and the $(4 \times 1)\left(\Theta_{\mathrm{Br}}=0.75 \mathrm{ML}\right)$ structure. In contrast to the short-bridge sites, it is possible to populate neighbouring long-bridge sites, which (due to lateral relaxation) gives rise to a $\mathrm{Br}-\mathrm{Br}$ distance of only $3.45 \AA$ within the troughs [24]. This demonstrates a large local variation of $\mathrm{Br}-\mathrm{Br}$ interactions with a particularly long-ranged repulsion on the close-packed atom rows. Note, that covalent binding has been found for this adsorbate system [18]. Hence direct $\mathrm{Br}-\mathrm{Br}$ interactions are expected to become important only for distances below twice the covalent $\mathrm{Br}$ radius (i.e. $\sim 2.2 \AA$ ). This is significantly lower than the length scales reported here. The $\mathrm{Br}-\mathrm{Br}$ interaction found in the present system is therefore indirect and mediated through the substrate electronic states. The particularly long-ranged repulsion on the close-packed rows is consequently attributable to electronic states which are confined to these rows.

In summary, the quasi-1D nature of the $c(2 \times 2)-$ $\mathrm{Br} / \mathrm{Pt}(110)$ system is documented by several independent observations. As expected for quasi-1D systems, one also finds rather unusual phase transitions, which will be described in the following section.

\section{Anomalous phase transition due to competing electronic interactions}

At room temperature a coverage of $\Theta_{\mathrm{Br}}=0.5 \mathrm{ML}$ results in a perfect $c(2 \times 2)$ LEED pattern. The only anomaly is the appearance of a strong Lorentzian component in the half-order spot profile taken along the [110] direction (Fig. 6). This reveals the presence of fluctuations, which are, however, coherent from row to row. Nevertheless, a large Gaussian contribution is found for the spot profiles in both directions, signalling a well developed long-range order. Cooling of the system to $50 \mathrm{~K}$ (the lowest temperature, which can be reached in our variable-temperature STM) destroys the long-range order completely. Instead a glassy phase develops with $c(2 \times 2)$ and $(2 \times 1)$ domains alternating randomly in [001] direction. Occasionally, $(3 \times 2)$ structural elements with 3 atoms per unit cell (yielding again a local coverage of $\Theta_{\mathrm{Br}}=0.5 \mathrm{ML}$ ) are observed [22]. On virtually defect-free preparations no $(3 \times 2)$ structural elements appear and the domains are extended over hundreds of Angstroms along the [110] direction. Further details will be reported elsewhere [25].

DFT calculations predict the $(2 \times 1)$ structure to be the stable groundstate at $T=0 \mathrm{~K}$, while the $c(2 \times 2)$ structure is $\sim 20 \mathrm{meV}$ higher in energy. Accordingly, the results seem to indicate an order-order transition from the $(2 \times 1)$ groundstate to a long-range ordered $c(2 \times 2)$ state at $300 \mathrm{~K}$, which sets in already below $50 \mathrm{~K}$. Order-order transitions as a function of temperature are rarely observed in adsorbate systems. To our knowledge, the one found for $\mathrm{CO}$ on $\mathrm{Co}(10 \overline{1} 0)$ by King and co-workers [26] is the only case, which has been analysed in detail. Here, a low- $T$ quasi-hexagonal CO packing is converted to a high$T$ zigzag phase at a critical temperature of about $150 \mathrm{~K}$. Below $100 \mathrm{~K}$ CO populates threefold hollow sites as well as short-bridge sites thus forming an almost hexagonal packing, which obviously minimises steric repulsion. In the $p(2 \times 1) g$ zigzag structure at $T>150 \mathrm{~K}$ the $\mathrm{CO}$ molecules populate short-bridge sites only (with a slight tilt due to the repulsion). The explanation for the phase transition in this case seems to be straightforward: the vibrational potential in the twofold site is much shallower than in the threefold site. Accordingly the vibrational wave number differs by almost an order of magnitude. Consequently, the higher vibrational entropy in the $p(2 \times 1) g$ state drives the phase transition. The present case is qualitatively different: the adsorbate-adsorbate repulsion is minimised in the high- $T$ as opposed to the low- $T$ phase. The vibrational entropy can be estimated from the potential energy surface determined via DFT calculations. It is slightly higher for 
the $c(2 \times 2)$ phase, because the absence of nearest neighbours across the rows leads to a slightly softer potential in that direction as compared to the $(2 \times 1)$ structure. The difference in estimated wave numbers, however, is less than a factor of two $\left(30 \mathrm{~cm}^{-1}\right.$ and $50 \mathrm{~cm}^{-1}$, resp.). Based on this estimate one would arrive at a critical temperature for the $(2 \times 1) \rightarrow c(2 \times 2)$ transition of more than $400 \mathrm{~K}$ in stark contrast to the observation of an almost balanced co-existence of the two phases already at $50 \mathrm{~K}$. An examination of possible models leads to the conclusion that the phase transition requires $T$-dependent interactions and/or considerable entropic contributions from the substrate. This is supported by the geometry of the two phases: in the $c(2 \times 2)$ phase the substrate remains essentially bulk terminated apart from a slight inward relaxation of the first Pt layer. In contrast, the $(2 \times 1)$ structure involves a considerable buckling of the second layer Pt atoms $(\sim 0.7 \AA)$. As mentioned before, circumstantial evidence indicates the presence of a $2 k_{F}$ interaction in the system $[21,27]$. We propose a CDW mechanism for the stabilization of the $(2 \times 1)$ structure (on perfectly prepared surfaces with $\Theta_{\mathrm{Br}} \leqslant 0.5 \mathrm{ML}$ exclusively the twofold periodicity appears, while the threefold periodicity seems to be preferred in the presence of defects and for Br coverages exceeding 0.5 ML). According to this model the $\mathrm{Pt}(110)$ surface is close to a Peierls instability. This originates from the existence of the saddle points at $E_{F}$ (see Fig. 3) with a connecting vector $q=2 k_{F} \approx 1.3 \AA^{-1}$, i.e. $1 / 2 G_{x}<q<2 / 3 G_{x}$. While no Peierls transition is known to occur on the bare surface, the adsorption of Br could actually trigger the transition, as the $\mathrm{Br}$ atoms lock into the CDW at sufficiently low temperature. This process can be modelled in the Landau formalism of phase transitions by introducing a coupling term between the order parameter $h$ of the CDW and the $\mathrm{Br}$ atoms. Since this interaction changes sign depending on whether the charge density at the Br sites is increased or decreased relative to the normal phase, the coupling term has to contain only odd powers of $h$. The leading term of the coupling is linear in $h$, thus having a similar effect as an external field in Landau theory. Higher odd powers in $h$ can be omitted at the present level of approximation, since they do not alter the basic physics anymore. A minimal model incorporating the essential interactions is therefore based on the following Hamiltonian:

$$
\frac{H}{A}=J s_{1} s_{2}+g h\left(s_{1}+s_{2}\right)+a h^{2}
$$

here we have assigned pseudo-spins $s_{i}, s_{j}$ to the shortbridge sites, which are \pm 1 depending on whether the sites are occupied $(+)$ or unoccupied $(-) . J$ is the repulsive energy between $\mathrm{Br}$ atoms in adjacent short-bridge sites across the rows. $h$ is the (dimensionless) order parameter of a CDW, e.g. the normalised amplitude of the lattice distortion. $a h^{2}$ is then the energy cost to establish a CDW phase in the bare substrate. A pivotal role is played by the middle term $g h\left(s_{1}+s_{2}\right)$, which introduces a coupling between the $\mathrm{Br}$ atoms and the CDW. Assuming without loss of generality $g<0$ one obtains for $s_{1}=s_{2}=1$ an energy gain due to the locking in of the Br overlayer on

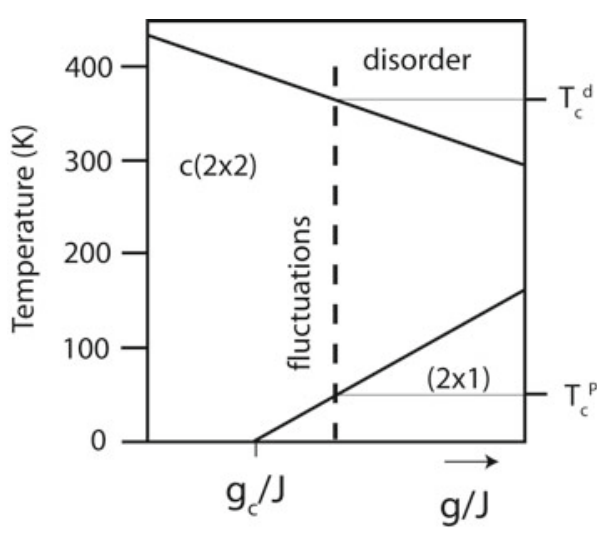

Fig. 7. Schematic phase diagram resulting from the minimal model according to equation (1).

the CDW-modulated substrate. At $T=0 \mathrm{~K}$ equation (1) yields for the $c(2 \times 2)$ phase $\left(s_{1}=-s_{2}\right)$ a minimum energy of $E_{c(2 \times 2)}=-J$, since the coupling term vanishes and the quadratic one is minimised by $h=0$. For the $(2 \times 1)$ structure $\left(s_{1}=s_{2}= \pm 1\right)$ the $h$-dependent interaction yields $a(h \pm g / a)^{2}-g^{2} / a$ and the groundstate energy is $E_{(2 \times 1)}=J-g^{2} / a$ corresponding to a finite lattice distortion $h_{0}= \pm g / a$. By a comparison of the energies for the two phases we find a critical coupling strength of $g_{c}=\sqrt{2 J a}$. For smaller $g$ the $c(2 \times 2)$ structure is favoured, due to the minimization of the repulsive energy. For larger $g$ the $(2 \times 1)$ phase is stabilized by the effective "external" field $h$, which jumps at the critical coupling strength from zero to $h_{0}=\sqrt{2 J / a}$. For a given $g>g_{c}$ one obtains the CDW stabilised $(2 \times 1)$ phase for $T<T_{c}^{\mathrm{P}}$, where $T_{c}^{\mathrm{P}}$ is the Peierls temperature of the system including the $\mathrm{Br}$ overlayer. The stability of the CDW phase obviously increases with the coupling strength $g$ and so does the Peierls temperature. By the same token the critical temperature for disordering the $c(2 \times 2)$ phase decreases, since the repulsive inter-adsorbate interaction $J$ stabilising the latter phase competes with the coupling of the adsorbate atoms to the charge density fluctuations in the substrate. Similarly, the effective Peierls temperature $T_{c}^{\mathrm{P}}$ is lower than the "bare" Peierls temperature due to the presence of the competing interaction $J$. The schematic phase diagram shown in Figure 7 is based on these considerations. The vertical dashed line describes the relevant trajectory for $0.5 \mathrm{ML}$ of $\mathrm{Br}$ on $\mathrm{Pt}(110)$. At $0 \mathrm{~K}$ the groundstate is $(2 \times 1)$. As the temperature is raised above the Peierls temperature, the $2 k_{F}$ interaction is suppressed, the CDW phase starts melting and the associated fluctuations drive the transition into the $c(2 \times 2)$ structure, which is stabilised by the virtually $T$-independent $\mathrm{Br}-\mathrm{Br}$ repulsion. Although the effective Peierls temperature is only about $50 \mathrm{~K}$ due to the competing $J$ interaction, CDW fluctuations survive up to much higher temperatures. Going beyond the minimal model represented by equation (1) one has to include a quartic term in $h$ to provide an upper boundary for the CDW order parameter. Furthermore, for an appropriate description of the fluctuations, spatial and temporal derivatives of the amplitude and phase of the 
CDW order parameter have to be added [28]. A more detailed discussion will be given elsewhere [25]. Finally, we briefly comment on the relation between the phase diagrams shown in Figures 1 and 7, respectively. The Peierls temperature $T_{c}^{\mathrm{P}}$ of Figure 7 corresponds to the mean field temperature $T_{M F}$ of Figure 1. The disordering temperature $T_{c}^{d}$ of Figure 7 is not related to the coherence temperature $T_{\text {coh }}$ of Figure 1, because the former does not relate to the coherence of the $(2 \times 1)$ phase, i.e. to the coherence of charge density fluctuations between individual 1D subunits. Rather it characterises the coherence of the $c(2 \times 2)$ phase, which is mainly determined by $J$.

In summary, we provide evidence for quasi-1D physics governing the behaviour of the $c(2 \times 2)-\operatorname{Br} / \operatorname{Pt}(110)$ system. This is due to the presence of numerous $d$ band derived surface resonances, which provide a substantial contribution to the DOS at $E_{F}$. Quasi-1D behaviour manifest itself not only in the strong anisotropy of electronic dispersions, inter-adsorbate interactions and fluctuation characteristics, but most prominently leads to an anomalous phase transition from a CDW stabilised phase into a quasi-hexagonal phase. Despite the weakly first-order character of the order-order phase transition, it is associated with quasi-critical fluctuations over a temperature range of $\sim 250 \mathrm{~K}$. In a strongly simplified model this can be qualitatively understood on the basis of a competition between indirect adsorbate-adsorbate repulsion and a tendency to form a Peierls distorted groundstate. The system should thus allow studying the dynamics of fluctuations over an extremely wide temperature window and associated range of time scales.

The work was supported by the Austrian Science Funds through NFN S9004 (NSOS).

\section{References}

1. T. Giamarchi, Quantum Physics in One Dimension (Oxford University Press, New York, 2004)

2. P. Monthoux, D. Pines, G.G. Lonzarich, Nature 450, 1177 (2007)

3. C. Hertlein, L. Helden, A. Gambassi, S. Dietrich, C. Bechinger, Nature 451, 172 (2008)

4. P. Coleman, A.J. Schofield, Nature 433, 226 (2005)

5. J. Schäfer, S. Meyer, C. Blumenstein, K. Roensch, Claessen, S. Mietke, M. Klinke, T. Podlich, R.
Matzdorf, A.A. Stekolnikov, S. Sauer, F. Bechstedt, http://arxiv.org/abs/0906.4912 (2009)

6. S. Riikonen, D. Sanchez-Portal, Phys. Rev. B (Condensed Matter and Materials Physics) 76, 035410 (2007)

7. H.W. Yeom, Phys. Rev. Lett. 97, 189701 (2006)

8. C. Gonzalez, F. Flores, J. Ortega, Phys. Rev. Lett. 97, $189702(2006)$

9. H.W. Yeom, S. Takeda, E. Rotenberg, I. Matsuda, K. Horikoshi, J. Schaefer, C.M. Lee, S.D. Kevan, T. Ohta, T. Nagao, S. Hasegawa, Phys. Rev. Lett. 82, 4898 (1999)

10. P.C. Snijders, S. Rogge, H.H. Weitering, Physi. Rev. Lett. 96, $076801(2006)$

11. M. Imada, A. Fujimori, Y. Tokura, Rev. Mod. Phys. 70, 1039 (1998)

12. W. Eberhardt, E.W. Plummer, K. Horn, J. Erskine, Phys. Rev. Lett. 45, 273 (1980)

13. G. Kresse, J. Furthmüller, Phys. Rev. B 54, 11169 (1996), http://cms.mpi.univie.ac.at/vasp/

14. Y. Wang, J.P. Perdew, Phys. Rev. B 44, 13298 (1991)

15. J.P. Perdew, A. Zunger, Phys. Rev. B 23, 5048 (1981)

16. D.M. Ceperley, B.J. Alder, Phys. Rev. Lett. 45, 566 (1980)

17. F. Tran, R. Laskowski, P. Blaha, K. Schwarz, Phys. Rev. B (Condensed Matter and Materials Physics) 75, 115131 (2007)

18. V. Blum, L. Hammer, K. Heinz, C. Franchini, J. Redinger, K. Swamy, C. Deisl, E. Bertel, Phys. Rev. B 65, 165408 (2002)

19. A. Menzel, Z. Zhang, M. Minca, T. Loerting, C. Deisl, E. Bertel, New J. Phys. 7, 102 (2005)

20. T.M. Rice, G.K. Scott, Phys. Rev. Lett. 35, 120 (1975)

21. C. Deisl, K. Swamy, N. Memmel, E. Bertel, C. Franchini, G. Schneider, J. Redinger, S. Walter, L. Hammer, K. Heinz, Phys. Rev. B 69, 195405 (2004)

22. E. Dona, T. Loerting, S. Penner, M. Minca, A. Menzel, E. Bertel, J. Schoiswohl, S. Berkebile, F.P. Netzer, R. Zucca, J. Redinger, Phys. Rev. Lett. 98, 186101 (2007)

23. M. Schick, Progress in Surface Science 11, 245 (1981)

24. C. Deisl, E. Dona, S. Penner, M. Gabl, E. Bertel, R. Zucca, J. Redinger, J. Phys.: Condens. Matt. 21, 134003 (2009)

25. M. Cordin, B. Lechner, P. Amann, A. Menzel, E. Bertel, R. Zucca, C. Franchini, J. Redinger, M. Baranov, S. Diehl, (submitted)

26. J. Gu, W.S. Sim, D.A. King, J. Chem. Phys. 107, 5613 (1997)

27. K. Swamy, A. Menzel, R. Beer, E. Bertel, Phys. Rev. Lett. 86, 1299 (2001)

28. G. Grüner, Density waves in solids (Perseus Publishing, Cambridge, MA, 1994) 